\title{
Controversies in the treatment of patients with STEMI and multivessel disease: is it time for PCI of all lesions?
}

\author{
Peter Ong $^{1} \cdot$ Udo Sechtem $^{1}$
}

Received: 16 November 2015/Accepted: 19 January 2016/Published online: 5 February 2016

(C) Springer-Verlag Berlin Heidelberg 2016

\begin{abstract}
Several randomized trials have suggested a benefit for multivessel PCI in patients with STEMI and multivessel disease. However, none of the studies compared multivessel PCI with a staged PCI-approach which is the current guideline recommended approach. The results of the trials may overestimate the beneficial effect of the multivessel PCI approach because the control group did not receive any ischaemia testing for evaluation of the significance of remaining lesions. Thus, unfavourable aspects of the multivessel PCI approach such as overestimation of non-culprit lesions at the time of acute coronary angiography, complications associated with PCI of the non-culprit lesion (i.e. dissection, no-reflow, acute stent thrombosis) or increased risk for contrast induced nephropathy may have gone unnoticed as the comparative management pathway was unusual and likely inferior to the guideline recommended approach. We believe that culprit lesion only PCI and staged evaluation of remaining areas of myocardial ischaemia with subsequent $\mathrm{PCI}$ is still preferable in patients with STEMI and multivessel disease but a randomized study comparing this approach with multivessel PCI is needed.
\end{abstract}

Keywords STEMI - Culprit lesion - Multivessel disease · FFR

Peter Ong

peter.ong@rbk.de

1 Department of Cardiology, Robert-Bosch-Krankenhaus, Auerbachstraße 110, 70376 Stuttgart, Germany

\section{Introduction}

In patients with ST-segment myocardial infarction (STEMI) primary percutaneous coronary intervention (PCI) of the culprit lesion is the treatment of choice [1]. However, it is known that approximately $50 \%$ of patients with STEMI have multivessel disease [2] and it has been shown that such patients have a worse outcome compared to patients with single vessel disease [3]. In addition, it is known that the presence of a chronic total occlusion in a non-infarct-related artery is an independent predictor of increased late mortality to 3 years [4]. Several approaches of dealing with the remaining lesions are possible: (1) one can perform PCI at the time of treating the culprit lesion based on the angiographic appearance of the stenoses, (2) one can perform PCI at the time of treating the culprit lesion based on a FFR measurement, (3) one can perform non-invasive (imaging) ischaemia testing and revascularise lesions perfusing ischaemic areas (the so-called staged procedure) and (4) one can leave the remaining lesions alone and act only if additional events occur (Fig. 1). Previous studies have shown that outcome of patients with STEMI and multivessel disease is better when a staged procedure is performed compared to unconditioned multivessel PCI at the time of primary PCI [5-7]. This has resulted in a class IIa recommendation for staged PCI in patients with STEMI and multivessel disease in the current guidelines by the European Society of Cardiology unless the patient presents with cardiogenic shock [1].

This recommendation has been challenged by the PRAMI trial published in 2013 [8]. In this multicenter study from the United Kingdom, 465 patients with STEMI were randomized in a 1:1 fashion to either multivessel PCI of all lesions $>50 \%$ (preventive PCI) or culprit lesion only (no preventive $\mathrm{PCI}$ ). There was a statistically significant 


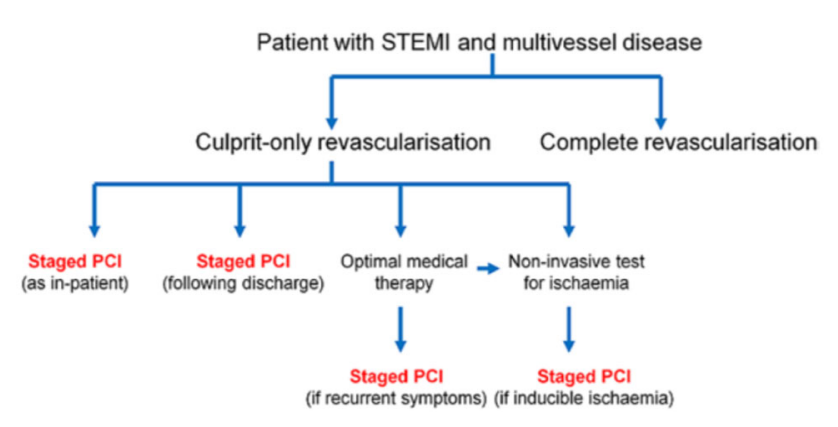

Fig. 1 Revascularisation strategies for ST-segment-elevation myocardial infarction (STEMI) patients with multivessel disease. $P C I$ indicated percutaneous coronary intervention (taken with permission from Ref. [7])

benefit for patients with preventive PCI regarding the primary outcome (combination of composite of death from cardiac causes, nonfatal myocardial infarction, or refractory angina) and also for the components nonfatal myocardial infarction and refractory angina. A strong tendency in favour of multivessel PCI was seen for death of cardiac causes. In addition, the secondary outcome, i.e. repeat revascularization, occurred significantly more often in the group without preventive PCI. In the CvLPRIT study [9], a multicenter trial from the United Kingdom, 296 patients with STEMI were randomized in a 1:1 fashion to either culprit lesion only PCI or multivessel PCI of all stenoses $>70 \%$. Complete revascularization was associated with a statistically significant benefit for the primary endpoint (combination of all-cause death, recurrent myocardial infarction, heart failure and ischaemia-driven revascularization within 12 months). The main point of criticism is that no ischaemia testing was performed as an inpatient nor following discharge in the control group leaving potentially critical lesions untreated.

A different approach was chosen in the recently published DANAMI-3-PRIMULTI trial [10]. In this Danish study 627 patients with STEMI and multivessel disease were randomized in a 1:1 fashion to either sole treatment of the culprit lesion with PCI or complete FFR-guided revascularization before discharge. Interestingly, about a third of patients initially assigned to complete revascularization based on visual angiographic criteria exhibited nonsignificant lesions based on FFR measurements and did not receive further PCI. Although no significant difference was found between the two groups for all-cause mortality and non-fatal reinfarction at a median of 27 months follow-up, patients in the FFR-guided revascularization group had significantly fewer repeat revascularizations. It should however be noted that the study was not powered to detect significant differences in mortality or the occurrence of reinfarction. Again, patients in the control group (culprit lesion PCI only) were potentially undertreated because no ischaemia testing was performed after the initial intervention.

The results of the studies addressed above have stimulated the discussion whether and when non-culprit lesions in patients with STEMI and multivessel disease should be treated. In contrast to some authors [11] who suggested that immediate multivessel PCI in such patients should be the default strategy, we strongly believe that all three studies (i.e. PRAMI, CVLPRIT, DANAMI-3-PRIMULTI) had a seriously flawed study design preventing meaningful conclusions regarding optimal treatment. The fault was that patients who received only PCI of the culprit lesion were not allowed to be treated according to the current guideline recommendation. The guidelines [1] call for staged revascularization after stabilization of the patient according to proof of myocardial ischaemia on further testing. However, in all three studies the study protocol demanded that patients in the control groups were treated in a suboptimal fashion (i.e. treatment according to the current guideline recommendation was not allowed). This may have favoured that all three trials showed statistically significant differences between the culprit lesion only group and the multivessel PCI group for the various endpoints. The fact that the staged approach recommended by the guidelines can still be regarded as the treatment of choice is supported by recent data from Israel showing that patients with STEMI and multivessel disease who underwent myocardial scintigraphy after primary PCI of the culprit lesion and were found to have no relevant ischaemia had a similar prognosis compared to patients with STEMI and single vessel disease [12].

There are some other potential pitfalls of the unconditioned multivessel PCI approach. First, the severity of nonculprit lesions at the time of acute angiography may be overestimated first because visual estimation of stenosis severity has a well-known imprecision and interobserver variability and second because lesions may be more severe in the acute situation due to additional vasospastic narrowing which is no longer present at control angiography a few weeks later [13]. However, there may also be nonischaemia inducing non-infarct related artery stenoses which may be more prone to plaque rupture or erosion in an activated, inflammatory ACS-milieu compared to a stable disease state with a potential impact on future major adverse events [14].

Second, complications associated with indiscriminate PCI of the non-culprit lesion (i.e. dissection, no-reflow, acute stent thrombosis) may lead to a worse outcome [15]. Third, there is an increased risk for contrast induced nephropathy. This is the reason why the guidelines recommend initially performing culprit lesion only PCI in patients with STEMI and multivessel disease unless they present with cardiogenic shock and have a subtotal stenosis 
in a non-culprit vessel. Two to three days after primary PCI one should perform stress imaging and subsequently perform PCI of lesions responsible for regional ischaemia. In the absence of ischaemia, the patient is managed conservatively. Most likely, addressing the hemodynamic significance of additional lesions using FFR during the initial emergency coronary intervention is another reasonable approach [9], but we do not yet have data comparing this approach to a staged strategy using stress imaging.

\section{Outlook}

There are several studies currently ongoing in this area. The COMPLETE trial is an international multicenter trial aiming at recruiting 3900 patients with end of enrolment envisaged in 2018. Patients with STEMI and multivessel disease are randomized to either complete revascularization (i.e. PCI of all lesions with a visual diameter stenosis of 70 or $50 \%$ visual with FFR $\leq 0.80$ ) within 45 days of the index PCI or culprit lesion only PCI. Again, it appears that this study is not allowing patients in the control group to receive staged revascularization according to assessment of myocardial ischaemia using non-invasive imaging techniques or FFR. In contrast, the currently ongoing CULPRIT-SHOCK trial enrolls patients with acute myocardial infarction, multivessel disease and cardiogenic shock. They will be randomized to either culprit lesion only PCI or immediate multivessel PCI of all lesions $>2 \mathrm{~mm}$ in diameter with at least $70 \%$ visual stenosis. The study aims at recruiting 706 patients in several European countries with an estimated primary completion date in December 2016. Interestingly, the study protocol apparently allows complete revascularization of the non-culprit lesions at a later time point as staged procedure depending on remaining ischaemia in the group that initially receives culprit lesion only PCI. The results of these and other ongoing studies will hopefully be helpful to decide upon the best treatment options in these patients.

\section{Conclusion}

Although several randomized studies have suggested a benefit for multivessel PCI in patients with STEMI and multivessel disease, none of the studies compared multivessel PCI with a staged PCI-approach according to the current guideline recommendations. Thus, we believe that culprit lesion only PCI and staged evaluation of remaining areas of myocardial ischaemia with subsequent PCI are still preferable in patients with STEMI and multivessel disease. This should, however, be proven in an appropriate randomized trial.
Compliance with ethical standards

Conflict of interest No conflicts to disclose.

\section{References}

1. Windecker S, Kolh P, Alfonso F, Collet JP, Cremer J, Falk V, Filippatos G, Hamm C, Head SJ, Jüni P, Kappetein AP, Kastrati A, Knuuti J, Landmesser U, Laufer G, Neumann FJ, Richter DJ, Schauerte P, Sousa Uva M, Stefanini GG, Taggart DP, Torracca L, Valgimigli M, Wijns W, Witkowski A (2014) ESC/EACTS Guidelines on myocardial revascularization: the Task Force on Myocardial Revascularization of the European Society of Cardiology (ESC) and the European Association for Cardio-Thoracic Surgery (EACTS)Developed with the special contribution of the European Association of Percutaneous Cardiovascular Interventions (EAPCI). Eur Heart J 2014(35):2541-2619

2. Goldstein JA, Demetriou D, Grines CL, Pica M, Shoukfeh M, O'Neill WW (2000) Multiple complex coronary plaques in patients with acute myocardial infarction. $\mathrm{N}$ Engl J Med 343:915-922

3. Sorajja P, Gersh BJ, Cox DA, McLaughlin MG, Zimetbaum P, Costantini C, Stuckey T, Tcheng JE, Mehran R, Lansky AJ, Grines CL, Stone GW (2007) Impact of multivessel disease on reperfusion success and clinical outcomes in patients undergoing primary percutaneous coronary intervention for acute myocardial infarction. Eur Heart J 28:1709-1716

4. Claessen BE, Dangas GD, Weisz G, Witzenbichler B, Guagliumi G, Möckel M, Brener SJ, Xu K, Henriques JP, Mehran R, Stone GW (2012) Prognostic impact of a chronic total occlusion in a non-infarct-related artery in patients with ST-segment elevation myocardial infarction: 3-year results from the HORIZONS-AMI trial. Eur Heart J 33:768-775

5. Kornowski R, Mehran R, Dangas G, Nikolsky E, Assali A, Claessen BE, Gersh BJ, Wong SC, Witzenbichler B, Guagliumi G, Dudek D, Fahy M, Lansky AJ (2011) Stone GW; HORIZONS-AMI Trial Investigators. Prognostic impact of staged versus "one-time" multivessel percutaneous intervention in acute myocardial infarction: analysis from the HORIZONS-AMI (harmonizing outcomes with revascularization and stents in acute myocardial infarction) trial. J Am Coll Cardiol 58:704-711

6. Hannan EL, Samadashvili Z, Walford G, Holmes DR Jr, Jacobs AK, Stamato NJ, Venditti FJ, Sharma S, King SB 3rd (2010) Culprit vessel percutaneous coronary intervention versus multivessel and staged percutaneous coronary intervention for STsegment elevation myocardial infarction patients with multivessel disease. JACC Cardiovasc Interv 3:22-31

7. Iqbal MB, Ilsley C, Kabir T, Smith R, Lane R, Mason M, Clifford P, Crake T, Firoozi S, Kalra S, Knight C, Lim P, Malik IS, Mathur A, Meier P, Rakhit RD, Redwood S, Whitbread M, Bromage D, Rathod K, MacCarthy P, Dalby M, London Heart Attack Centre (HAC) Group Investigators (2014) Culprit vessel versus multivessel intervention at the time of primary percutaneous coronary intervention in patients with ST-segment-elevation myocardial infarction and multivessel disease: real-world analysis of 3984 patients in London. Circ Cardiovasc Qual Outcomes 7:936-943

8. Wald DS, Morris JK, Wald NJ, Chase AJ, Edwards RJ, Hughes LO, Berry C, Oldroyd KG, PRAMI Investigators (2013) Randomized trial of preventive angioplasty in myocardial infarction. N Engl J Med 369:1115-1123

9. Gershlick AH, Khan JN, Kelly DJ, Greenwood JP, Sasikaran T, Curzen N, Blackman DJ, Dalby M, Fairbrother KL, Banya W, Wang D, Flather M, Hetherington SL, Kelion AD, Talwar S, 
Gunning M, Hall R, Swanton H, McCann GP (2015) Randomized trial of complete versus lesion-only revascularization in patients undergoing primary percutaneous coronary intervention for STEMI and multivessel disease: the CvLPRIT trial. J Am Coll Cardiol 65:963-972

10. Engstrøm T, Kelbæk H, Helqvist S, Høfsten DE, Kløvgaard L, Holmvang L, Jørgensen E, Pedersen F, Saunamäki K, Clemmensen P, De Backer O, Ravkilde J, Tilsted HH, Villadsen AB, Aarøe J, Jensen SE, Raungaard B, Køber L, DANAMI-3-PRIMULTI Investigators (2015) Complete revascularisation versus treatment of the culprit lesion only in patients with ST-segment elevation myocardial infarction and multivessel disease (DANAMI-3-PRIMULTI): an open-label, randomised controlled trial. Lancet 386:665-671

11. Moretti C, D'Ascenzo F, Quadri G, Omedè P, Montefusco A, Taha S, Cerrato E, Colaci C, Chen SL, Biondi-Zoccai G, Gaita F (2015) Management of multivessel coronary disease in STEMI patients: a systematic review and meta-analysis. Int J Cardiol 179:552-557

12. Weissler-Snir A, Gurevitz C, Assali A, Vaknin-Assa H, Bental T, Lador A, Yavin H, Perl L, Kornowski R, Lev E (2015) Prognosis of STEMI patients with multi-vessel disease undergoing culpritonly PCI without significant residual ischemia on non-invasive stress testing. PLoS One 10:e0138474

13. Hanratty CG, Koyama Y, Rasmussen HH, Nelson GI, Hansen PS, Ward MR (2002) Exaggeration of nonculprit stenosis severity during acute myocardial infarction: implications for immediate multivessel revascularization. J Am Coll Cardiol 40:911-916

14. Stone GW, Maehara A, Lansky AJ, de Bruyne B, Cristea E, Mintz GS, Mehran R, McPherson J, Farhat N, Marso SP, Parise $\mathrm{H}$, Templin B, White R, Zhang Z, Serruys PW, PROSPECT Investigators (2011) A prospective natural-history study of coronary atherosclerosis. N Engl J Med 364:226-235

15. Toma M, Buller CE, Westerhout CM, Fu Y, O'Neill WW, Holmes DR Jr, Hamm CW, Granger CB, Armstrong PW, APEXAMI Investigators (2010) Non-culprit coronary artery percutaneous coronary intervention during acute ST-segment elevation myocardial infarction: insights from the APEX-AMI trial. Eur Heart J 31:1701-1707 